\title{
Measurement of the $\mathrm{W}^{+} \mathrm{W}^{-} \gamma$ cross section and direct limits on anomalous quartic gauge boson couplings at LEP
}

\section{L3 Collaboration}

M. Acciarri ${ }^{\mathrm{z}}$, P. Achard $^{\mathrm{s}}$, O. Adriani ${ }^{\mathrm{p}}, \mathrm{M}$. Aguilar-Benitez ${ }^{\mathrm{y}}, \mathrm{J}$. Alcaraz $^{\mathrm{y}}$, G. Alemanni ${ }^{\mathrm{v}}$, J. Allaby ${ }^{\mathrm{q}}$, A. Aloisio ${ }^{\mathrm{ab}}$, M.G. Alviggi ${ }^{\mathrm{ab}}$, G. Ambrosi ${ }^{\mathrm{s}}$, H. Anderhub av , V.P. Andreev f,aj, T. Angelescu ${ }^{1}$, F. Anselmo ${ }^{\mathrm{i}}$, A. Arefiev ${ }^{\text {aa }}$, T. Azemoon ${ }^{\text {c }}$, T. Aziz ${ }^{\text {j }}$ P. Bagnaia ${ }^{\text {ai }}$, A. Bajo ${ }^{\mathrm{y}}$, L. Baksay $^{\mathrm{aq}}$, A. Balandras ${ }^{\mathrm{d}}$, S.V. Baldew ${ }^{b}$, S. Banerjee ${ }^{j}$, Sw. Banerjee ${ }^{j}$, A. Barczyk ${ }^{\text {av,at }}$, R. Barillère ${ }^{q}$, P. Bartalini ${ }^{v}$, M. Basile ${ }^{i}$, R. Battiston ${ }^{\text {af }}$, A. Bay ${ }^{\mathrm{v}}$, F. Becattini ${ }^{\mathrm{p}}$, U. Becker ${ }^{\mathrm{n}}$, F. Behner ${ }^{\text {av }}$, L. Bellucci ${ }^{\mathrm{p}}$, R. Berbeco ${ }^{\mathrm{c}}$, J. Berdugo ${ }^{\mathrm{y}}$, P. Berges ${ }^{\mathrm{n}}$, B. Bertucci ${ }^{\text {af }}$, B.L. Betev ${ }^{\text {av }}$, S. Bhattacharya ${ }^{j}$, M. Biasini ${ }^{\text {af }}$, A. Biland ${ }^{\text {av }}$, J.J. Blaising ${ }^{d}$, S.C. Blyth ${ }^{\text {ag }}$, G.J. Bobbink ${ }^{\text {b }}$, A. Böhm ${ }^{\text {a }}$, L. Boldizsar ${ }^{\mathrm{m}}$, B. Borgia ${ }^{\text {ai }}$, D. Bourilkov $^{\text {av }}$, M. Bourquin ${ }^{\text {s, S. Braccini }}{ }^{\text {s }}$, J.G. Branson ${ }^{\mathrm{am}}$, F. Brochu ${ }^{\mathrm{d}}$, A. Buffini $^{\mathrm{p}}$, A. Buijs ${ }^{\text {ar }}$, J.D. Burger ${ }^{\mathrm{n}}$, W.J. Burger ${ }^{\text {af }}$, X.D. Cai ${ }^{\mathrm{n}}$, M. Capell ${ }^{\mathrm{n}}$, G. Cara Romeo ${ }^{\mathrm{i}}$, G. Carlino ${ }^{\mathrm{ab}}$, A.M. Cartacci ${ }^{\mathrm{p}}$, J. Casaus ${ }^{\mathrm{y}}$, G. Castellini $^{\mathrm{p}}$, F. Cavallari ${ }^{\text {ai }}$, N. Cavallo ${ }^{\text {ak }}$, C. Cecchi ${ }^{\text {af }}$, M. Cerrada ${ }^{\mathrm{y}}$, F. Cesaroni ${ }^{\mathrm{w}}, \mathrm{M}$. Chamizo ${ }^{\mathrm{s}}$, Y.H. Chang ${ }^{\text {ax }}$, U.K. Chaturvedi ${ }^{\mathrm{r}}$, M. Chemarin ${ }^{\mathrm{x}}$, A. Chen ${ }^{\mathrm{ax}}$, G. Chen ${ }^{\mathrm{g}}$, G.M. Chen ${ }^{\mathrm{g}}$, H.F. Chen ${ }^{t}$, H.S. Chen ${ }^{g}$, G. Chiefari ${ }^{\text {ab }}$, L. Cifarelli ${ }^{\text {al }}$, F. Cindolo ${ }^{i}$, C. Civinini ${ }^{p}$, I. Clare ${ }^{\mathrm{n}}$, R. Clare $^{\mathrm{n}}$, G. Coignet ${ }^{\mathrm{d}}$, N. Colino ${ }^{\mathrm{y}}$, S. Costantini ${ }^{\mathrm{e}}$, F. Cotorobai ${ }^{1}$, B. de la Cruz ${ }^{\mathrm{y}}$, A. Csilling ${ }^{\mathrm{m}}$, S. Cucciarelli ${ }^{\text {af }}$, T.S. Dai ${ }^{\mathrm{n}}$, J.A. van Dalen ${ }^{\text {ad }}$, R. D'Alessandro ${ }^{p}$, R. de Asmundis ${ }^{a b}$, P. Déglon ${ }^{\mathrm{s}}$, A. Degré ${ }^{\mathrm{d}}, \mathrm{K}$. Deiters ${ }^{\text {at }}$, D. della Volpe ${ }^{\mathrm{ab}}$, E. Delmeire ${ }^{\mathrm{s}}$, P. Denes ${ }^{\text {ah }}$, F. DeNotaristefani ${ }^{\text {ai }}$, A. De Salvo ${ }^{\text {av }}$, M. Diemoz ai ${ }^{\text {ai }}$ M. Dierckxsens ${ }^{\text {b }}$, D. van Dierendonck ${ }^{\text {b }}$, C. Dionisi ${ }^{\text {ai }}$, M. Dittmar ${ }^{\text {av }}$, A. Dominguez ${ }^{\text {am }}$, A. Doria ${ }^{\text {ab }}$, M.T. Dova ${ }^{\mathrm{r}, 5}$, D. Duchesneau ${ }^{\mathrm{d}}$, D. Dufournaud ${ }^{\mathrm{d}}$, P. Duinker ${ }^{\text {b }}$, I. Duran ${ }^{\text {an }}$, H. El Mamouni ${ }^{\mathrm{x}}$, A. Engler ${ }^{\mathrm{ag}}$, F.J. Eppling ${ }^{\text {n }}$, F.C. Erné ${ }^{\mathrm{b}}$,

P. Extermann ${ }^{\text {s }}$, M. Fabre ${ }^{\text {at }}$, M.A. Falagan ${ }^{\mathrm{y}}$, S. Falciano ${ }^{\text {q,ai }}$, A. Favara ${ }^{\mathrm{q}}$, J. Fay ${ }^{\mathrm{x}}$, O. Fedin ${ }^{\text {aj }}$, M. Felcini ${ }^{\text {av }}$, T. Ferguson ${ }^{\text {ag }}$, H. Fesefeldt ${ }^{\text {a }}$, E. Fiandrini ${ }^{\text {af }}$, J.H. Field ${ }^{\mathrm{s}}$, F. Filthaut ${ }^{\mathrm{q}}$, P.H. Fisher ${ }^{\mathrm{n}}$, I. Fisk ${ }^{\text {am }}$, G. Forconi ${ }^{\mathrm{n}}$, K. Freudenreich ${ }^{\text {av }}$, C. Furetta ${ }^{\mathrm{z}}$, Yu. Galaktionov ${ }^{\text {aa, }}$, S.N. Ganguli ${ }^{\text {', P. Garcia-Abia }}{ }^{\text {e }}$, M. Gataullin ${ }^{\text {ae }}$, S.S. Gau ${ }^{\text {, }}$, S. Gentile ${ }^{\text {ai, }}$, N. Gheordanescu ${ }^{1}$, S. Giagu ${ }^{\text {ai }}$, Z.F. Gong ${ }^{\text {t }}$, G. Grenier ${ }^{\text {x }}$, O. Grimm ${ }^{\text {av }}$, 
M.W. Gruenewald ${ }^{\text {h }}$, M. Guida ${ }^{\text {al }}$, R. van Gulik ${ }^{b}$, V.K. Gupta ${ }^{\text {ah }}$, A. Gurtu ${ }^{j}$, L.J. Gutay ${ }^{\text {as }}$, D. Haas ${ }^{\text {e }}$, A. Hasan ${ }^{\text {ac }}$, D. Hatzifotiadou ${ }^{\text {i, T. Hebbeker }}{ }^{\text {h }}$, A. Hervé ${ }^{q}$, P. Hidas ${ }^{\mathrm{m}}$, J. Hirschfelder ${ }^{\text {ag }}$, H. Hofer ${ }^{\text {av }}$, G. Holzner ${ }^{\text {av }}$, H. Hoorani ${ }^{\text {ag }}$, S.R. Hou ${ }^{\text {ax }}$, Y. Hu ${ }^{\text {ad }}$, I. Iashvili ${ }^{\text {au }}$, B.N. Jin ${ }^{\mathrm{g}}$, L.W. Jones ${ }^{\text {c }}$, P. de Jong ${ }^{\mathrm{b}}$, I. Josa-Mutuberría ${ }^{\mathrm{y}}$, R.A. Khan ${ }^{\text {r }}$, M. Kaur ${ }^{\text {r,6 }}$, M.N. Kienzle-Focacci ${ }^{\text {s }}$, D. Kim ${ }^{\text {ai }}$, J.K. Kim ${ }^{\text {ap }}$, J. Kirkby ${ }^{\text {q }}$, D. Kiss ${ }^{\text {m }}$, W. Kittel ${ }^{\text {ad }}$, A. Klimentov ${ }^{\text {n,aa }}$, A.C. König ${ }^{\text {ad }}$, A. Kopp ${ }^{\text {au }}$, V. Koutsenko ${ }^{\text {n,aa }}$, M. Kräber ${ }^{\text {av }}$, R.W. Kraemer ${ }^{\text {ag }}$, W. Krenz ${ }^{\text {a }}$, A. Krüger ${ }^{\text {au }}$, A. Kunin ${ }^{\text {n,aa }}$, P. Ladron de Guevara ${ }^{y}$, I. Laktineh ${ }^{\mathrm{x}}$, G. Landi ${ }^{\mathrm{p}}$, M. Lebeau ${ }^{\mathrm{q}}$, A. Lebedev ${ }^{n}$, P. Lebrun ${ }^{x}$, P. Lecomte ${ }^{\text {av }}$, P. Lecoq ${ }^{q}$, P. Le Coultre ${ }^{\text {av }}$, H.J. Lee ${ }^{\text {h }}$, J.M. Le Goff ${ }^{\mathrm{q}}$, R. Leiste ${ }^{\text {au }}$, P. Levtchenko ${ }^{\text {aj }},{\text { C. } \text { Li }{ }^{\mathrm{t}}, \text { S. Likhoded }}^{\text {au }}$, C.H. Lin ${ }^{\text {ax }}$,

W.T. Lin ${ }^{a x}$, F.L. Linde ${ }^{b}$, L. Lista ${ }^{\text {ab }}$, Z.A. Liu ${ }^{g}$, W. Lohmann ${ }^{\text {au }}$, E. Longo ${ }^{\text {ai }}$, Y.S. Lu ${ }^{g}$, K. Lübelsmeyer ${ }^{\text {a }}$, C. Luci ${ }^{\text {q,ai }}$, D. Luckey ${ }^{n}$, L. Lugnier ${ }^{x}$, L. Luminari ${ }^{\text {ai }}$,

W. Lustermann ${ }^{\text {av }}$, W.G. Ma ${ }^{\mathrm{t}}$, M. Maity ${ }^{\mathrm{j}}$, L. Malgeri ${ }^{\mathrm{q}}$, A. Malinin ${ }^{\mathrm{q}}$, C. Maña ${ }^{\mathrm{y}}$, D. Mangeol ad ${ }^{\text {a }}$ J. Mans ${ }^{\text {ah }}$, G. Marian ${ }^{\text {, }}$, J.P. Martin ${ }^{\text {x }}$, F. Marzano ai, K. Mazumdar ${ }^{\mathrm{j}}$, R.R. McNeil ${ }^{\text {f }}$, S. Mele ${ }^{\mathrm{q}}$, L. Merola ${ }^{\text {ab }}$, M. Meschini ${ }^{\mathrm{p}}$, W.J. Metzger ${ }^{\text {ad }}$, M. von der Mey ${ }^{\text {a }}$, A. Mihul ${ }^{1}$, H. Milcent ${ }^{\mathrm{q}}$, G. Mirabelli ${ }^{\text {ai }}$, J. Mnich ${ }^{\mathrm{q}}$, G.B. Mohanty ${ }^{\text {j }}$, T. Moulik ${ }^{j}$, G.S. Muanza ${ }^{x}$, A.J.M. Muijs ${ }^{b}$, B. Musicar ${ }^{a m}$, M. Musy ${ }^{\text {ai }}$, M. Napolitano ${ }^{\text {ab }}$, F. Nessi-Tedaldi ${ }^{\text {av }}$, H. Newman ${ }^{\text {ae }}$, T. Niessen ${ }^{\text {a }}$, A. Nisati ${ }^{\text {ai }}$, H. Nowak ${ }^{\text {au }}$, R. Ofierzynski ${ }^{\text {av }}$, G. Organtini ${ }^{\text {ai }}$, A. Oulianov ${ }^{\text {aa }}$, C. Palomares ${ }^{\mathrm{y}}$, D. Pandoulas ${ }^{\mathrm{a}}, \mathrm{S}$. Paoletti ${ }^{\text {q,ai }}$, P. Paolucci ${ }^{\text {ab }}$, R. Paramatti ${ }^{\text {ai }}$, H.K. Park ${ }^{\text {ag }}$, I.H. Park ${ }^{\text {ap }}$, G. Passaleva ${ }^{\mathrm{q}}$, S. Patricelli ${ }^{\text {ab }}$, T. Paul ${ }^{\mathrm{k}}$, M. Pauluzzi ${ }^{\text {af }}$, C. Paus ${ }^{\mathrm{q}}$, F. Pauss ${ }^{\text {av }}$, M. Pedace ${ }^{\text {ai }}$, S. Pensotti ${ }^{z}$, D. Perret-Gallix ${ }^{\text {d }}$, B. Petersen ${ }^{\text {ad }}$, D. Piccolo $^{\text {ab }}$, F. Pierella ${ }^{\text {i }}$, M. Pieri ${ }^{\text {p }}$, P.A. Piroué ${ }^{\text {ah }}$, E. Pistolesi ${ }^{z}$, V. Plyaskin ${ }^{\text {aa }}$, M. Pohl ${ }^{\text {s }}$, V. Pojidaev aa, ${ }^{\text {a }}$ H. Postema ${ }^{n}$, J. Pothier ${ }^{\text {q }}$, D.O. Prokofiev ${ }^{\text {as }}$, D. Prokofiev aj, J. Quartieri ${ }^{\text {al }}$, G. Rahal-Callot ${ }^{\text {av,q }}$, M.A. Rahaman ${ }^{j}$, P. Raics ${ }^{\circ}$, N. Raja ${ }^{\mathrm{j}}$, R. Ramelli ${ }^{\text {av }}$, P.G. Rancoita ${ }^{\mathrm{z}}$, R. Ranieri ${ }^{\mathrm{p}}$, A. Raspereza ${ }^{\mathrm{au}}$, G. Raven $^{\mathrm{am}}{ }^{\text {, }}$ P. Razis ${ }^{\text {ac }}$, D. Ren ${ }^{\text {av }}$, M. Rescigno ${ }^{\text {ai }}$, S. Reucroft ${ }^{\text {k }}$, S. Riemann ${ }^{\text {au }}$, K. Riles ${ }^{c}$, J. Rodin ${ }^{\text {aq }}$, B.P. Roe ${ }^{\mathrm{c}}$, L. Romero ${ }^{\mathrm{y}}$, A. Rosca ${ }^{\mathrm{h}}$, S. Rosier-Lees ${ }^{\mathrm{d}}$, J.A. Rubio ${ }^{\mathrm{q}}$, G. Ruggiero $^{\text {p }}$, H. Rykaczewski ${ }^{\text {av }}$, S. Saremi ${ }^{\text {f }}$, S. Sarkar ${ }^{\text {ai }}$, J. Salicio ${ }^{\text {q }}$, E. Sanchez ${ }^{\text {q }}$, M.P. Sanders ${ }^{\text {ad }}$, M.E. Sarakinos ${ }^{\text {u }}$, C. Schäfer ${ }^{\text {q }}$, V. Schegelsky aj, S. Schmidt-Kaerst ${ }^{\text {a }}$, D. Schmitz ${ }^{\text {a }}$, H. Schopper ${ }^{\text {aw }}$, D.J. Schotanus ${ }^{\text {ad }}$, G. Schwering ${ }^{\text {a }}$, C. Sciacca ${ }^{\text {ab }}$, A. Seganti ${ }^{\text {i }}$, L. Servoli ${ }^{\text {af }}$, S. Shevchenko ${ }^{\text {ae }}$, N. Shivarov ${ }^{\text {ao }}$, V. Shoutko ${ }^{\text {aa }}$, E. Shumilov ${ }^{\text {aa }}$, A. Shvorob ${ }^{\text {ae }}$, T. Siedenburg ${ }^{\text {a }}$, D. Son ${ }^{\text {ap }}$, B. Smith ${ }^{\text {ag }}$, P. Spillantini ${ }^{\text {, }}$, M. Steuer ${ }^{n}$, D.P. Stickland ${ }^{\text {ah }}$, A. Stone ${ }^{\text {f }}$, B. Stoyanov ${ }^{\text {ao }}$, A. Straessner ${ }^{\text {a }}$, K. Sudhakar ${ }^{\mathrm{j}}$, G. Sultanov ${ }^{\mathrm{r}}$, L.Z. Sun ${ }^{\mathrm{t}}$, H. Suter ${ }^{\text {av }}$, J.D. Swain ${ }^{\mathrm{r}}$, Z. Szillasi ${ }^{\text {aq,3, }}$, T. Sztaricskai aq,3 X.W. Tang ${ }^{\mathrm{g}}$, L. Tauscher ${ }^{\mathrm{e}}$, L. Taylor ${ }^{\mathrm{k}}$, B. Tellili ${ }^{\mathrm{x}}$, C. Timmermans ${ }^{\text {ad }}$, Samuel C.C. Ting ${ }^{n}$, S.M. Ting ${ }^{n}$, S.C. Tonwar ${ }^{j}$, J. Tóth ${ }^{\mathrm{m}}$, C. Tully ${ }^{\mathrm{q}}$, K.L. Tung ${ }^{\mathrm{g}}$, Y. Uchida ${ }^{\mathrm{n}}$, J. Ulbricht ${ }^{\text {av }}$, E. Valente ${ }^{\text {ai }}$, G. Vesztergombi ${ }^{\mathrm{m}}$, 
I. Vetlitsky $^{\text {aa }}$, D. Vicinanza ${ }^{\text {al }}$, G. Viertel ${ }^{\text {av }}$, S. Villa ${ }^{\text {k }}$, P. Violini ${ }^{\mathrm{q}}$, M. Vivargent ${ }^{\mathrm{d}}$, S. Vlachos ${ }^{\text {e }}$, I. Vodopianov ${ }^{\text {aj }}$, H. Vogel ${ }^{\text {ag }}$, H. Vogt ${ }^{\text {au }}$, I. Vorobiev ${ }^{\text {aa }}$, A.A. Vorobyov ${ }^{\text {aj }}$, A. Vorvolakos ${ }^{\text {ac }}$, M. Wadhwa ${ }^{\mathrm{e}}$, W. Wallraff ${ }^{\mathrm{a}}$, M. Wang ${ }^{\mathrm{n}}$, X.L. Wang ${ }^{\mathrm{t}}$, Z.M. Wang ${ }^{\mathrm{t}}$, A. Weber ${ }^{\mathrm{a}}$, M. Weber ${ }^{\mathrm{a}}$, P. Wienemann ${ }^{\mathrm{a}}$, H. Wilkens ${ }^{\text {ad }}$, S.X. Wu ${ }^{\mathrm{n}}$, S. Wynhoff ${ }^{\mathrm{q}}, \mathrm{L} \mathrm{Xia}^{\mathrm{ae}}$, Z.Z. Xu ${ }^{\mathrm{t}}$, J. Yamamoto $^{\mathrm{c}}$, B.Z. Yang ${ }^{\mathrm{t}}$, C.G. Yang ${ }^{\mathrm{g}}$, H.J. Yang ${ }^{\mathrm{g}}$, M. Yang ${ }^{\mathrm{g}}$, J.B. Ye ${ }^{\mathrm{t}}$, S.C. Yeh ${ }^{\text {ay }}$, An. Zalite ${ }^{\text {aj, }}$, Yu. Zalite ${ }^{\text {aj }}$, Z.P. Zhang ${ }^{\text {t }}$, G.Y. Zhu ${ }^{\mathrm{g}}$, R.Y. Zhu ${ }^{\text {ae }}$, A. Zichichi ${ }^{\text {i,q,r }}$, G. Zilizi ${ }^{\text {aq,3, }}$, B. Zimmermann ${ }^{\text {av }}$, M. Zöller ${ }^{\text {a }}$

${ }^{a}$ I. Physikalisches Institut, RWTH, D-52056 Aachen, Germany, and III. Physikalisches Institut, RWTH, D-52056 Aachen, Germany ${ }^{I}$

${ }^{\mathrm{b}}$ National Institute for High Energy Physics, NIKHEF, and University of Amsterdam, NL-1009 DB Amsterdam, The Netherlands

c University of Michigan, Ann Arbor, MI 48109, USA

${ }^{\mathrm{d}}$ Laboratoire d'Annecy-le-Vieux de Physique des Particules, LAPP, IN2P3-CNRS, BP 110, F-74941 Annecy-le-Vieux CEDEX, France

e Institute of Physics, University of Basel, CH-4056 Basel, Switzerland

${ }^{\mathrm{f}}$ Louisiana State University, Baton Rouge, LA 70803, USA

g Institute of High Energy Physics, IHEP, 100039 Beijing, China ${ }^{7}$

${ }^{\mathrm{h}}$ Humboldt University, D-10099 Berlin, Germany ${ }^{1}$

${ }^{\mathrm{i}}$ University of Bologna and INFN-Sezione di Bologna, I-40126 Bologna, Italy

${ }^{\mathrm{j}}$ Tata Institute of Fundamental Research, Bombay 400 005, India

${ }^{\mathrm{k}}$ Northeastern University, Boston, MA 02115, USA

${ }^{1}$ Institute of Atomic Physics and University of Bucharest, R-76900 Bucharest, Romania

${ }^{\mathrm{m}}$ Central Research Institute for Physics of the Hungarian Academy of Sciences, H-1525 Budapest 114, Hungary ${ }^{2}$

${ }^{\mathrm{n}}$ Massachusetts Institute of Technology, Cambridge, MA 02139, USA

${ }^{\circ}$ KLTE-ATOMKI, H-4010 Debrecen, Hungary ${ }^{3}$

${ }^{\mathrm{p}}$ INFN Sezione di Firenze and University of Florence, I-50125 Florence, Italy

${ }^{\mathrm{q}}$ European Laboratory for Particle Physics, CERN, CH-1211 Geneva 23, Switzerland

${ }^{\mathrm{r}}$ World Laboratory, FBLJA Project, CH-1211 Geneva 23, Switzerland

${ }^{\mathrm{s}}$ University of Geneva, CH-1211 Geneva 4, Switzerland

${ }^{\mathrm{t}}$ Chinese University of Science and Technology, USTC, Hefei, Anhui 230 029, China ${ }^{7}$

" SEFT, Research Institute for High Energy Physics, P.O. Box 9, SF-00014 Helsinki, Finland

${ }^{\vee}$ University of Lausanne, CH-1015 Lausanne, Switzerland

${ }^{\mathrm{w}}$ INFN-Sezione di Lecce and Universitá Degli Studi di Lecce, I-73100 Lecce, Italy

${ }^{x}$ Institut de Physique Nucléaire de Lyon, IN2P3-CNRS, Université Claude Bernard, F-69622 Villeurbanne, France

${ }^{y}$ Centro de Investigaciones Energéticas, Medioambientales y Tecnologícas, CIEMAT, E-28040 Madrid, Spain ${ }^{4}$

z INFN-Sezione di Milano, I-20133 Milan, Italy

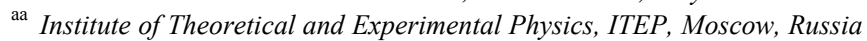

${ }^{\mathrm{ab}}$ INFN-Sezione di Napoli and University of Naples, I-80125 Naples, Italy

${ }^{\text {ac }}$ Department of Natural Sciences, University of Cyprus, Nicosia, Cyprus

${ }^{\text {ad }}$ University of Nijmegen and NIKHEF, NL-6525 ED Nijmegen, The Netherlands

ae California Institute of Technology, Pasadena, CA 91125, USA

af INFN-Sezione di Perugia and Universitá Degli Studi di Perugia, I-06100 Perugia, Italy

${ }^{\text {ag }}$ Carnegie Mellon University, Pittsburgh, PA 15213, USA

ah Princeton University, Princeton, NJ 08544, USA

ai INFN-Sezione di Roma and University of Rome, "La Sapienza", I-00185 Rome, Italy

${ }^{\text {aj }}$ Nuclear Physics Institute, St. Petersburg, Russia

ak INFN-Sezione di Napoli and University of Potenza, I-85100 Potenza, Italy

${ }^{\text {al }}$ University and INFN, Salerno, I-84100 Salerno, Italy

am University of California, San Diego, CA 92093, USA

${ }^{a n}$ Dept. de Fisica de Particulas Elementales, Univ. de Santiago, E-15706 Santiago de Compostela, Spain

ao Bulgarian Academy of Sciences, Central Lab. of Mechatronics and Instrumentation, BU-1113 Sofia, Bulgaria

${ }^{\text {ap }}$ Laboratory of High Energy Physics, Kyungpook National University, 702-701 Taegu, South Korea

${ }^{\text {aq }}$ University of Alabama, Tuscaloosa, AL 35486, USA

${ }^{\text {ar }}$ Utrecht University and NIKHEF, NL-3584 CB Utrecht, The Netherlands

as Purdue University, West Lafayette, IN 47907, USA 


\author{
${ }^{\text {at }}$ Paul Scherrer Institut, PSI, CH-5232 Villigen, Switzerland \\ ${ }^{\text {au }}$ DESY, D-15738 Zeuthen, Germany \\ av Eidgenössische Technische Hochschule, ETH Zürich, CH-8093 Zürich, Switzerland \\ aw University of Hamburg, D-22761 Hamburg, Germany \\ ${ }^{a x}$ National Central University, Chung-Li, Taiwan, ROC \\ ay Department of Physics, National Tsing Hua University, Taiwan, ROC
}

Received 19 July 2000; accepted 11 August 2000

Editor: K. Winter

\begin{abstract}
The process $\mathrm{e}^{+} \mathrm{e}^{-} \rightarrow \mathrm{W}^{+} \mathrm{W}^{-} \gamma$ is analysed using the data collected with the L3 detector at LEP at a centre-of-mass energy of $188.6 \mathrm{GeV}$, corresponding to an integrated luminosity of $176.8 \mathrm{pb}^{-1}$. Based on a sample of 42 selected $\mathrm{W}^{+} \mathrm{W}^{-}$ candidates containing an isolated hard photon, the $\mathrm{W}^{+} \mathrm{W}^{-} \gamma$ cross section, defined within phase-space cuts, is measured to be: $\sigma_{\mathrm{WW} \gamma}=290 \pm 80 \pm 16 \mathrm{fb}$, consistent with the Standard Model expectation. Including the process $\mathrm{e}^{+} \mathrm{e}^{-} \rightarrow \nu \bar{\nu} \gamma \gamma$, limits are derived on anomalous contributions to the Standard Model quartic vertices $\mathrm{W}^{+} \mathrm{W}^{-} \gamma \gamma$ and $\mathrm{W}^{+} \mathrm{W}^{-} \mathrm{Z} \gamma$ at $95 \% \mathrm{CL}$ : $-0.043 \mathrm{GeV}^{-2}<a_{0} / \Lambda^{2}<0.043 \mathrm{GeV}^{-2}, \quad-0.08 \mathrm{GeV}^{-2}<a_{c} / \Lambda^{2}<0.13 \mathrm{GeV}^{-2}, \quad-0.41 \mathrm{GeV}^{-2}<a_{n} / \Lambda^{2}<0.37 \mathrm{GeV}^{-2}$. (C) 2000 Elsevier Science B.V. All rights reserved.
\end{abstract}

\section{Introduction}

The LEP centre-of-mass energy for $\mathrm{e}^{+} \mathrm{e}^{-}$collisions is now well above the kinematic threshold for $\mathrm{W}$-pair production allowing for the study of radiative $\mathrm{W}$-pair production, $\mathrm{e}^{+} \mathrm{e}^{-} \rightarrow \mathrm{W}^{+} \mathrm{W}^{-} \gamma$. The Standard Model (SM) [1,2] predicts the existence of quartic gauge couplings (QGCs), leading to $\mathrm{W}^{+} \mathrm{W}^{-} \gamma$ production via $s$-channel exchange of a $\gamma$ or $\mathrm{Z}$ boson as shown in Fig. 1a.

As the contribution of these two quartic Feynman diagrams with respect to the other competing diagrams, mainly initial-state radiation, is negligible at the LEP centre-of-mass energies, the process leading

\footnotetext{
${ }^{1}$ Supported by the German Bundesministerium für Bildung, Wissenschaft, Forschung und Technologie.

${ }^{2}$ Supported by the Hungarian OTKA fund under contract numbers T019181, F023259 and T024011.

${ }^{3}$ Also supported by the Hungarian OTKA fund under contract numbers T22238 and T026178.

${ }^{4}$ Supported also by the Comisión Interministerial de Ciencia y Tecnología.

${ }^{5}$ Also supported by CONICET and Universidad Nacional de La Plata, CC 67, 1900 La Plata, Argentina.

${ }^{6}$ Also supported by Panjab University, Chandigarh-160014, India.

${ }^{7}$ Supported by the National Natural Science Foundation of China.
}

to the $\mathrm{W}^{+} \mathrm{W}^{-} \gamma$ final state could thus be sensitive to anomalous contributions to the SM quartic gauge-boson vertices $\mathrm{W}^{+} \mathrm{W}^{-} \gamma \gamma$ and $\mathrm{W}^{+} \mathrm{W}^{-} \mathrm{Z} \gamma$.

The existence of Anomalous QGCs (AQGCs) would also affect the $\mathrm{e}^{+} \mathrm{e}^{-} \rightarrow \nu_{\mathrm{e}} \overline{\mathrm{e}}_{\mathrm{e}} \gamma \gamma$ process via the $\mathrm{W}^{+} \mathrm{W}^{-}$fusion Feynman diagram containing the $\mathrm{W}^{+} \mathrm{W}^{-} \gamma \gamma$ vertex [3] (see Fig. 1b). In the SM the reaction $\mathrm{e}^{+} \mathrm{e}^{-} \rightarrow \nu \bar{\nu} \gamma \gamma$ proceeds predominantly through $s$-channel $\mathrm{Z}$ exchange and $t$-channel $\mathrm{W}$ exchange, with the two photons coming from initial state radiation, whereas the SM contribution from the $\mathrm{W}^{+} \mathrm{W}^{-}$fusion is negligible at LEP. AQGCs would enhance the $\nu \bar{\nu} \gamma \gamma$ production rate, especially for the hard tail of the photon energy distribution and for photons produced at large angles with respect to the beam direction.

Here we describe the cross section measurement for the process $\mathrm{e}^{+} \mathrm{e}^{-} \rightarrow \mathrm{W}^{+} \mathrm{W}^{-} \gamma$ and the determination of AQGCs using the data collected in 1998 with the L3 detector [4] at $\sqrt{s}=188.6 \mathrm{GeV}$ (denoted as $\sqrt{s}=189 \mathrm{GeV}$ hereafter) corresponding to an integrated luminosity of $176.8 \mathrm{pb}^{-1}$. AQGCs are also independently determined using acoplanar photonpair events with missing energy. This analysis is performed using the data at $\sqrt{s}=189 \mathrm{GeV}$ and at $\sqrt{s}=182.7 \mathrm{GeV}$ collected in 1997 (denoted as $\sqrt{\mathrm{s}}=$ $183 \mathrm{GeV}$ hereafter) corresponding to a total integrated luminosity of $231.7 \mathrm{pb}^{-1}$. The results derived 
a)

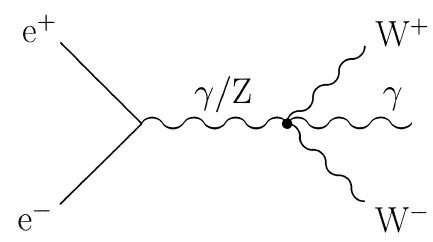

b)

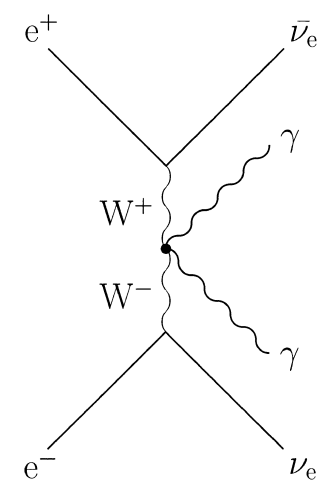

Fig. 1. Feynman diagrams containing a four-boson vertex leading to the (a) $\mathrm{W}^{+} \mathrm{W}^{-} \gamma$ and to the (b) $\nu_{\mathrm{e}} \overline{\mathrm{e}}_{\mathrm{e}} \gamma \gamma$ final states.

on AQGCs from the $\mathrm{W}^{+} \mathrm{W}^{-} \gamma$ and $\nu \bar{\nu} \gamma \gamma$ channels are finally combined.

The search for anomalous contributions to the SM quartic couplings is performed within the theoretical framework of Refs. [5,6]. Recently, experimental measurements for these couplings have already been performed on final states with three vector bosons $\mathrm{W}^{+} \mathrm{W}^{-} \gamma[7]$ and $\mathrm{Z} \gamma \gamma$ [8].

\section{2. $\mathrm{W}^{+} \mathrm{W}^{-} \gamma$ final state and signal definition}

There are 14 Feynman diagrams at the tree level leading to the $\mathrm{W}^{+} \mathrm{W}^{-} \gamma$ final state, and many other diagrams corresponding to photons from the decay products of hadronic or leptonic W's. We are interested only in two of these, the quartic diagrams. The other diagrams leading to the same final state are initial state radiation (ISR), final state radiation (FSR), and radiation from the $\mathrm{W}$ boson itself.

The Monte Carlo used for the $\mathrm{W}^{+} \mathrm{W}^{-} \gamma$ cross section determination is KORALW [9]. This generator does not include the quartic coupling diagrams. Initial state multi-photon radiation is implemented in KORALW in the full photon phase space. FSR from charged leptons in the event up to double bremsstrahlung is included using the PHOTOS [10] package. Fragmentation processes of quarks into hadrons are made according to the JETSET [11] algorithm including photons in the parton shower. For the $\mathrm{W}^{+} \mathrm{W}^{-} \gamma$ cross section measurement, this modelling is sufficient since the contribution of all the other diagrams is very small. The background processes such as $\mathrm{e}^{+} \mathrm{e}^{-} \rightarrow \mathrm{Z} / \gamma \rightarrow \mathrm{q} \overline{\mathrm{q}}(\gamma)$ and $\mathrm{e}^{+} \mathrm{e}^{-}$ $\rightarrow \mathrm{ZZ} \rightarrow 4 f(\gamma)$ are simulated using PYTHIA [12]. The L3 detector response is simulated by the program GEANT [13].

In this analysis, the $\mathrm{W}^{+} \mathrm{W}^{-} \gamma$ signal is defined by the following phase-space cuts:

- $E_{\gamma}>5 \mathrm{GeV}$, where $E_{\gamma}$ is the energy of the photon,

- $\theta_{\gamma}>20^{\circ}$, where $\theta_{\gamma}$ is the angle between the photon and the beam axis,

- $\alpha_{\gamma}>20^{\circ}$, where $\alpha_{\gamma}$ is the angle between the direction of the photon and that of the closest charged lepton or jet.

These cuts are mainly chosen for experimental reasons, to optimise the photon identification and the background suppression. They also largely avoid any infrared and collinear singularities in the calculation of the signal cross section.

The theoretically predicted $\mathrm{W}^{+} \mathrm{W}^{-} \gamma$ cross section from KORALW corresponds to $272 \pm 4$ (stat) $\mathrm{fb}$. In the EEWWG program [6] the effect of undetected additional ISR collinear to the beam pipe is included [14] by implementing the EXCALIBUR [15] collinear radiator function. The effect of the higher order radiative corrections is to move the effective centre-of-mass energy towards lower values, reducing the expected signal cross section by about $18 \%$. The resulting EEWWG cross section corresponds to $233 \pm 12$ (theor) $\mathrm{fb}$. This is used as the SM expectation in the anomalous coupling analysis, which leads to less stringent constraints on AQGCs. The theoretical uncertainty [14] is propagated to the AQGC determination. Consistent results are obtained with the YFSWW3 [16] MC which predicts $224 \pm 6$ (stat) fb. Differences of this order in the predicted cross section with high transverse momentum photons are expected [17] between pure leading-log and leading-log plus matrix-element based calculations. 


\section{3. $\mathrm{W}^{+} \mathrm{W}^{-} \gamma$ event selection and cross section}

The W-pair event selections used here are similar to those reported in Ref. [18]. Only the semileptonic and fully hadronic W-pair decay modes are considered. The number of selected data events and the expected number of signal and background events are shown in Table 1.

The photon selection in $\mathrm{W}^{+} \mathrm{W}^{-}$events is optimised for each four-fermion final state. Photons are selected by requiring energy deposition in the electromagnetic calorimeter not associated with any track in the central detector, and low hadronic activity in a cone of half-opening angle of $7^{\circ}$ around the electromagnetic cluster. The profile of the shower must be consistent with that of an electromagnetic particle. In addition, the highest energy photon has to satisfy the signal definition requirements: $E_{\gamma}>5 \mathrm{GeV}, \theta_{\gamma}>$ $20^{\circ}$, and $\alpha_{\gamma}>20^{\circ}$. Fig. 2 shows the distributions of $E_{\gamma}, \theta_{\gamma}$, and $\alpha_{\gamma}$, where for the last variable the direction of the reconstructed hadronic jet is assumed as the direction of the quark in the final state. In general, good agreement between the data and the SM expectation is observed.

Table 2 summarises the results for the applied selection criteria. In total $42 \mathrm{~W}^{+} \mathrm{W}^{-} \gamma$ events are selected, where $37.8 \pm 0.6$ is the Monte Carlo expectation. The efficiency $\varepsilon_{\mathrm{WW} \gamma}$ is defined as the number of selected KORALW events (regardless of any phase-space cuts) divided by the number of generated MC events satisfying the signal definition. It accounts for small possible migration effects of events from outside the signal region into the selected sample due to the finite detector resolution.

Table 1

Number of observed events, selection efficiencies with statistical uncertainties, expected total number of events and background estimates for the various $\mathrm{W}^{+} \mathrm{W}^{-}$decay channels according to the $\mathrm{SM}$ prediction. The efficiencies shown here include the contribution of cross efficiencies from the other W-pair decay modes.

\begin{tabular}{lclrc}
\hline Decay channel & $N_{\text {obs }}$ & $\varepsilon_{\mathrm{WW}}$ & $N_{\mathrm{TOT}}^{\exp }$ & $N_{\mathrm{Bkgr}}^{\text {exp }}$ \\
\hline qqe $\nu_{e}$ & 355 & $0.768 \pm 0.005$ & 361 & 19 \\
qq $\mu \nu_{\mu}$ & 364 & $0.834 \pm 0.002$ & 375 & 19 \\
qq $\tau \nu_{\tau}$ & 313 & $0.605 \pm 0.003$ & 300 & 42 \\
qqqq & 1514 & $0.892 \pm 0.006$ & 1486 & 296
\end{tabular}
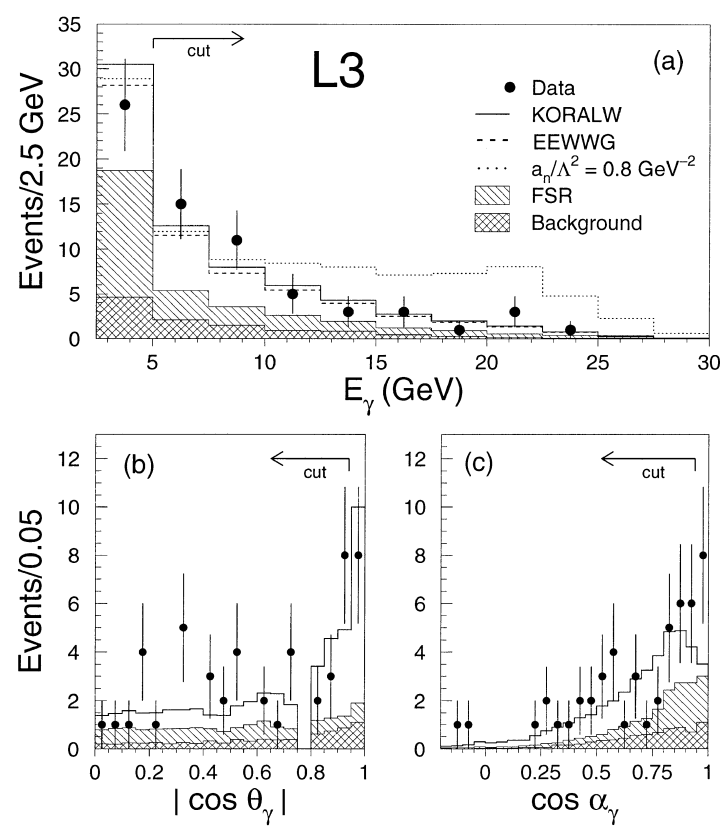

Fig. 2. Differential distributions, for the semileptonic and fully hadronic $\mathrm{W}^{+} \mathrm{W}^{-} \gamma$ decay modes, of (a) the photon energy, (b) the angle of the photon to the beam axis and (c) angle of the photon to the closest charged lepton or jet. The hatched area is the background component from ZZ, Zee, and $q \bar{q}(\gamma)$ events. Final state radiation includes the contribution of photons radiated off the charged fermions and photons originating from isolated meson decays. In the upper plot, the distribution corresponding to a non-zero value of the anomalous coupling $a_{n} / \Lambda^{2}$ is shown as a dotted line.

The $\mathrm{W}^{+} \mathrm{W}^{-} \gamma$ cross section is evaluated channel by channel and then combined according to the SM $\mathrm{W}$-pair branching fractions. The result is:

$\sigma_{\mathrm{WW} \gamma}=290 \pm 80 \pm 16 \mathrm{fb}$,

where the first error is statistical and the second systematic. The measurement is in good agreement

Table 2

Number of observed events, selection efficiencies with statistical uncertainties and expected number of total and background events including final state radiation.

\begin{tabular}{lccll}
\hline Decay channel & $N_{\mathrm{obs}}$ & $\varepsilon_{\mathrm{WW} \gamma}$ & $N_{\mathrm{TOT}}^{\exp }$ & $N_{\mathrm{FSR}}^{\exp }+N_{\mathrm{Bkgr}}^{\exp }$ \\
\hline qqe $\nu_{e} \gamma$ & 6 & $0.483 \pm 0.025$ & $5.85 \pm 0.26$ & $2.26 \pm 0.14$ \\
$\mathrm{qq} \mu \nu_{\mu} \gamma$ & 5 & $0.547 \pm 0.027$ & $6.87 \pm 0.28$ & $2.88 \pm 0.17$ \\
$\mathrm{qq} \tau \nu_{\tau} \gamma$ & 7 & $0.351 \pm 0.018$ & $4.63 \pm 0.22$ & $2.05 \pm 0.14$ \\
qqqq $\gamma$ & 24 & $0.504 \pm 0.016$ & $20.4 \pm 0.38$ & $9.23 \pm 0.26$ \\
total & 42 & - & $37.8 \pm 0.6$ & $16.4 \pm 0.4$ \\
\hline
\end{tabular}


with both the KORALW and EEWWG SM expectations.

Fig. 3 shows the result obtained together with the predicted total $\mathrm{W}^{+} \mathrm{W}^{-} \gamma$ cross section from the EEWWG Monte Carlo as a function of the centreof-mass energy.

The systematic uncertainties arising in the inclusive W-pair event selections [18] are propagated to the final measurement and correspond to an uncertainty of $\pm 6.3 \mathrm{fb}$. Other possible systematic biases due to detector effects such as electro-magnetic cluster resolution, angular resolution, and calorimetric energy scale uncertainty are found to have a negligible effect on the final result.

The total systematic uncertainty is dominated by the JETSET modelling of photons from meson decays $\left(\pi^{0}, \eta\right)$. To estimate this effect, a data sample of $3.9 \mathrm{pb}^{-1}$ collected in 1998 at $\sqrt{s}=91 \mathrm{GeV}$ is studied. The same photon identification criteria are applied to the selected $\mathrm{Z} \rightarrow \mathrm{q} \overline{\mathrm{q}}$ events. An overall excess of $(20 \pm 10) \%$ in the photon rate is found in data with respect to the PYTHIA Monte Carlo which uses the same JETSET fragmentation algorithm and particle decays as KORALW. A correction factor given by the ratio of photon production rates in data and $\mathrm{MC}$ is determined as a function of the photon energy. This correction is applied to the background component of $\mathrm{q} \overline{\mathrm{q}} \gamma \mathrm{MC}$ events as well as to the

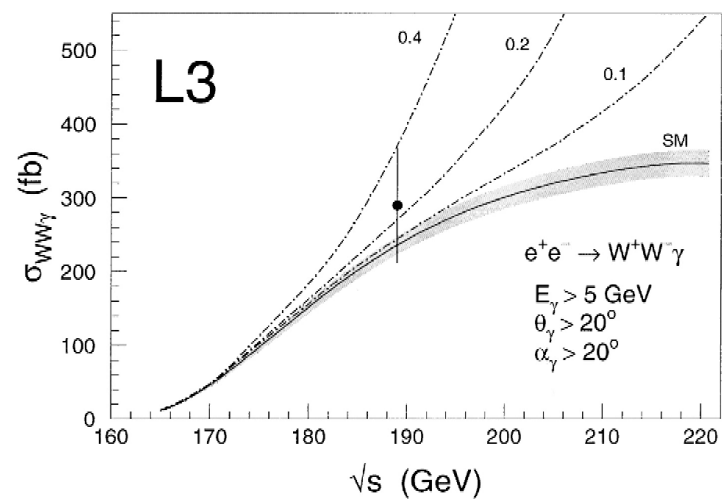

Fig. 3. Measured cross section for $\mathrm{e}^{+} \mathrm{e}^{-} \rightarrow \mathrm{W}^{+} \mathrm{W}^{-} \gamma$ at $\sqrt{s}=$ $189 \mathrm{GeV}$ (point) compared to the SM cross section as a function of the centre-of-mass energy (solid line) as predicted by the EEWWG Monte Carlo within the indicated phase-space cuts. The shaded band corresponds to the theoretical uncertainty of $\pm 5 \%$. The three dashed lines correspond to the cross section for nonvanishing values of the anomalous coupling $a_{n} / \Lambda^{2}$ (in $\mathrm{GeV}^{-2}$ units). hadronic side of the $\mathrm{W}^{+} \mathrm{W}^{-} \gamma \mathrm{MC}$ events. The uncertainty on this correction is propagated to the measurement as a systematic uncertainty on the $\mathrm{W}^{+} \mathrm{W}^{-} \gamma$ cross section which corresponds to \pm 15 $\mathrm{fb}$.

\section{Determination of anomalous quartic gauge cou- plings}

The selected $\mathrm{W}^{+} \mathrm{W}^{-} \gamma$ events allow us to constrain anomalous contributions to the SM quartic gauge boson vertices. In the framework of Refs. $[5,6]$, the extended Lagrangian includes new dimension- 6 operators,

$\mathscr{L}_{0}=-\frac{e^{2}}{16} \frac{a_{0}}{\Lambda^{2}} F^{\mu \nu} F_{\mu \nu} \boldsymbol{W}^{\alpha} \cdot \boldsymbol{W}_{\alpha}$

$\mathscr{L}_{c}=-\frac{e^{2}}{16} \frac{a_{c}}{\Lambda^{2}} F^{\mu \alpha} F_{\mu \beta} \boldsymbol{W}^{\beta} \cdot \boldsymbol{W}_{\alpha}$

$\mathscr{L}_{n}=-\frac{i e^{2}}{16} \frac{a_{n}}{\Lambda^{2}} \epsilon_{i j k} W_{\mu \alpha}^{(i)} W_{\nu}^{(j)} W^{(k) \alpha} F^{\mu \nu}$,

where $a_{0} / \Lambda^{2}, a_{c} / \Lambda^{2}$, and $a_{n} / \Lambda^{2}$ are the AQGCs, and $\Lambda$ represents the energy scale for new physics. The two parameters $a_{0} / \Lambda^{2}$ and $a_{c} / \Lambda^{2}$, which are separately $\mathrm{C}$ and $\mathrm{P}$ conserving, generate anomalous $\mathrm{W}^{+} \mathrm{W}^{-} \gamma \gamma$ and $\mathrm{ZZ} \gamma \gamma$ vertices. The term $a_{n} / \Lambda^{2}$, which is $\mathrm{CP}$ violating, gives rise to an anomalous contribution to $\mathrm{W}^{+} \mathrm{W}^{-} \mathrm{Z} \gamma$. Although there are already direct [7,8] and indirect [19] limits on $a_{0} / \Lambda^{2}$ and $a_{c} / \Lambda^{2}$, only the study of $\mathrm{W}^{+} \mathrm{W}^{-} \gamma$ events allows for a direct measurement of the anomalous coupling $a_{n} / \Lambda^{2}$ through the $\mathrm{W}^{+} \mathrm{W}^{-} \mathrm{Z} \gamma$ vertex.

The EEWWG program implements the effects of the AQGCs through the extended SM Lagrangian. Fig. 3 shows how the anomalous coupling $a_{n} / \Lambda^{2}$ manifests itself through a deviation of the total cross section.

The anomalous component from the above operators is linear in the photon energy at the matrix element level [6]. This implies that also the shape of the photon spectrum is affected by AQGCs, in particular, the hard part of the energy distribution (see Fig. 2a). The expected distribution for any value of the three AQGCs is obtained by reweighting each KORALW MC event with the ratio $\mathscr{W}\left(E_{\gamma}, a_{0}, a_{c}, a_{n}\right)$ 
of the known differential distributions of $\mathrm{E}_{\gamma}$ at generator level:

$$
\begin{aligned}
\mathscr{W} & \left(E_{\gamma}, a_{0}, a_{c}, a_{n}\right) \\
= & \frac{d \sigma^{\text {EEWWG }}}{d E_{\gamma}}\left(a_{0}, a_{c}, a_{n}\right) / \frac{d \sigma^{\text {KORALW }}}{d E_{\gamma}} .
\end{aligned}
$$

The reweighting procedure is applied only to the ISR component of the MC selected sample, while the FSR (from KORALW) and the background components of accepted events are kept fixed. The possible dependence of the selection efficiency on the photon polar angle and on the angular separation from the charged fermions in the event is found to be negligible.

Both the shape and the normalisation of the observed photon spectrum in the range from $5 \mathrm{GeV}$ to $30 \mathrm{GeV}$ are used in a maximum-likelihood fit to each of the anomalous couplings $a_{0} / \Lambda^{2}, a_{c} / \Lambda^{2}$ and $a_{n} / \Lambda^{2}$, fixing the other two to zero. The effects of the same systematic uncertainties described for the cross section measurement are included, yielding the $68 \% \mathrm{CL}$ intervals:

$$
\begin{aligned}
-0.028 \mathrm{GeV}^{-2} & <a_{0} / \Lambda^{2}<0.028 \mathrm{GeV}^{-2} \\
-0.04 \mathrm{GeV}^{-2} & <a_{c} / \Lambda^{2}<0.09 \mathrm{GeV}^{-2} \\
-0.26 \mathrm{GeV}^{-2} & <a_{n} / \Lambda^{2}<0.23 \mathrm{GeV}^{-2} .
\end{aligned}
$$

The results are in good agreement with the SM value of zero for each of the anomalous quartic gauge couplings. The 1-parameter limits at $95 \% \mathrm{CL}$ are:

$\begin{aligned}-0.045 \mathrm{GeV}^{-2} & <a_{0} / \Lambda^{2}<0.045 \mathrm{GeV}^{-2} \\ -0.08 \mathrm{GeV}^{-2} & <a_{c} / \Lambda^{2}<0.13 \mathrm{GeV}^{-2} \\ -0.41 \mathrm{GeV}^{-2} & <a_{n} / \Lambda^{2}<0.37 \mathrm{GeV}^{-2} .\end{aligned}$

\section{The $\mathrm{e}^{+} \mathrm{e}^{-} \rightarrow \nu \bar{\nu} \gamma \gamma$ process}

The selection of acoplanar multi-photon events is identical to that described in Ref. [20]. At least two photons with energies greater than $5 \mathrm{GeV}$ and $1 \mathrm{GeV}$ are required, with polar angles between $14^{\circ}$ and $166^{\circ}$. The KORALZ [21] and NUNUGPV [22] Monte Carlo generators are used to model the $\mathrm{e}^{+} \mathrm{e}^{-} \rightarrow \nu \bar{\nu} \gamma \gamma$ process according to the SM. The effects of the
AQGCs $a_{0} / \Lambda^{2}$ and $a_{c} / \Lambda^{2}$ are simulated using the EENUNUGGANO program [3]. Note that $\nu \bar{\nu} \gamma \gamma$ production is not sensitive to the $a_{n} / \Lambda^{2}$ coupling.

We select 14 events at $\sqrt{s}=183 \mathrm{GeV}$ and 21 events at $\sqrt{s}=189 \mathrm{GeV}$ compared to a SM expectation of 13.3 and 36.2 events respectively.

The EENUNUGGANO program does not describe the effects of the SM $s$-channel $Z$ exchange diagrams and the interference between these diagrams and the $\mathrm{W}^{+} \mathrm{W}^{-}$fusion diagram containing the $\mathrm{W}^{+} \mathrm{W}^{-} \gamma \gamma$ vertex. Therefore additional cuts are applied to suppress the SM contribution. The energy of both photons must be greater than $10 \mathrm{GeV}$. If both photons are in the barrel region $(|\cos \theta|<0.7)$, either the recoil mass must be less than $80 \mathrm{GeV}$ or the sum of the photon energies must be greater than 100 $\mathrm{GeV}$. If one or two photons are in the endcaps, where the SM contribution is larger, the recoil mass must be less than $75 \mathrm{GeV}$. After applying these cuts no data event is selected, consistent with the SM expectation of 0.15 events.

The expected number of events for any AQGC value is calculated based on a sample of ten thousand simulated EENUNUGGANO events generated for several values of $a_{0} / \Lambda^{2}$ and $a_{c} / \Lambda^{2}$. Its matrix element is used to reweight the events to any AQGC value required, testing the procedure by comparing the reweighted distributions to those from samples generated at various values of AQGCs. In all cases good agreement is observed.

Since the program does not include higher order corrections due to ISR, these effects are estimated by implementing the EXCALIBUR [15] collinear radiator function. The cross section is reduced by about $16 \%$ which is used in the following. The remaining theoretical uncertainty of 5\% [14] is taken into account in the AQGC limits. The systematic uncertainty on the selection efficiency [20] gives a much smaller contribution.

The 95\% CL upper limit on the number of expected events from the AQGC signal is obtained taking into account the systematic error on the accepted cross section; this corresponds to the following 1-parameter limits at 95\% CL:

$$
\begin{aligned}
-0.067 \mathrm{GeV}^{-2} & <a_{0} / \Lambda^{2}<0.066 \mathrm{GeV}^{-2} \\
-0.18 \mathrm{GeV}^{-2} & <a_{c} / \Lambda^{2}<0.18 \mathrm{GeV}^{-2} .
\end{aligned}
$$




\section{Conclusion}

All results obtained results are in a good agreement with the SM expectation of zero for each anomalous quartic gauge boson couplings.

Combining the results on $a_{0} / \Lambda^{2}$ and $a_{c} / \Lambda^{2}$ from our analyses of $\mathrm{W}^{+} \mathrm{W}^{-} \gamma$ and $\nu \bar{\nu} \gamma \gamma$ production, we derive the following 1-parameter 95\% CL limits:

$$
\begin{aligned}
-0.043 \mathrm{GeV}^{-2} & <a_{0} / \Lambda^{2}<0.043 \mathrm{GeV}^{-2} \\
-0.08 \mathrm{GeV}^{-2} & <a_{c} / \Lambda^{2}<0.13 \mathrm{GeV}^{-2} \\
-0.41 \mathrm{GeV}^{-2} & <a_{n} / \Lambda^{2}<0.37 \mathrm{GeV}^{-2} .
\end{aligned}
$$

\section{Acknowledgements}

We thank J.W. Stirling and A. Werthenbach for providing us with the $\mathrm{e}^{+} \mathrm{e}^{-} \rightarrow \mathrm{W}^{+} \mathrm{W}^{-} \gamma$ and the $\mathrm{e}^{+} \mathrm{e}^{-} \rightarrow \nu \bar{\nu} \gamma \gamma$ analytical calculations including AQGCs. We wish to express our gratitude to the CERN accelerator divisions for the superb performance and the continuous and successful upgrade of the LEP machine. We acknowledge the contributions of the engineers and technicians who have participated in the construction and maintenance of this experiment.

\section{References}

[1] S.L. Glashow, Nucl. Phys. 22 (1961) 579; A. Salam, in: N. Svartholm (Ed.), Elementary Particle Theory, Almqvist and Wiksell, Stockholm, 1968, p. 367; S. Weinberg, Phys. Rev. Lett. 19 (1967) 1264.

[2] M. Veltman, Nucl. Phys. B 7 (1968) 637; G.M.'t Hooft, Nucl. Phys. B 35 (1971) 167; G.M.'t Hooft, M. Veltman, Nucl. Phys. B 44 (1972) 189; Nucl. Phys. B 50 (1972) 318.
[3] J.W. Stirling, A. Werthenbach, Phys. Lett. B 466 (1999) 369.

[4] L3 Collaboration, B. Adeva et al., Nucl. Instr. Meth. A 289 (1990) 35; J.A. Bakken et al., Nucl. Instr. Meth. A 275 (1989) 81; O. Adriani et al., Nucl. Instr. Meth. A 302 (1991) 53; B. Adeva et al., Nucl. Instr. Meth. A 323 (1992) 109; K. Deiters et al., Nucl. Instr. Meth. A 323 (1992) 162; M. Chemarin et al., Nucl. Instr. Meth. A 349 (1994) 345; M. Acciarri et al., Nucl. Instr. Meth. A 351 (1994) 300; G. Basti et al., Nucl. Instr. Meth. A 374 (1996) 293; A. Adam et al., Nucl. Instr. Meth. A 383 (1996) 342.

[5] G. Belanger, F. Boudjema, Nucl. Phys. B 288 (1992) 201.

[6] J.W. Stirling, A. Werthenbach, Eur. Phys. J. C 14 (2000) 103.

[7] Opal Collaboration, G. Abbiendi et al., Phys. Lett. B 471 (1999) 293.

[8] L3 Collaboration, M. Acciarri et al., Phys. Lett. B 478 (2000) 34.

[9] KORALW V1.42, M. Skrzypek et al., Comp. Phys. Comm. 94 (1996) 216; Phys. Lett. B 372 (1996) 289.

[10] E. Barberio, B. van Eijk, Z. Was, Comp. Phys. Comm. 79 (1994) 291

[11] T. Sjöstrand, H.U. Bengtsson, Comp. Phys. Comm. 46 (1987) 43.

[12] T. Sjöstrand, Comp. Phys. Comm. 82 (1994) 74.

[13] The L3 detector simulation is based on GEANT Version 3.15. See R. Brun et al., GEANT 3, CERN DD/EE/84-1 (Revised), September 1987. The GHEISHA program (H. Fesefeldt, RWTH Aachen Report PITHA 85/02 (1985)) is used to simulate hadronic interactions.

[14] J.W. Stirling, A. Werthenbach, private communication.

[15] F.A. Berends, R. Pittau, R. Kleiss, Comp. Phys. Comm. 85 (1995) 437.

[16] S. Jadach et al., preprint UTHEP-00-0101, to appear.

[17] S. Jadach, private communication.

[18] L3 Collaboration, M. Acciarri et al., Preprint CERN-EP2000-104 (2000).

[19] O.J.P. Eboli, M.C. Gonzales-Garcia, Phys. Lett. B 411 (1994) 381

[20] L3 Collaboration, M. Acciarri et al., Phys. Lett. B 444 (1998) 503; Phys. Lett. B 470 (1999) 268.

[21] The KORALZ version 4.03 is used. S. Jadach, B.F.L. Ward, Z. Was, Comp. Phys Comm. 79 (1994) 503.

[22] G. Montagna et al., Nucl. Phys. B 541 (1999) 31. 